\title{
A Mobile Context-Aware System to Support People with the Retinitis Pigmentosa Disease
}

\author{
Edson D. M. Santos, Flávio D.F. Júnior, \\ José O. Ferreira, Talles M. de A. Barbosa \\ Department of Computing \\ Pontifical Catholic University of Goiás, PUC-Goiás \\ Goiânia, Brazil
}

\author{
Adson F. da Rocha \\ Department of Computing \\ UnB Gama Faculty, FGA \\ Brasília, Brazil
}

\begin{abstract}
Retinitis Pigmentosa (RP) is an eye disease that affects 1 in 4.000 people and it causes the loss of peripheral vision in early stages, possibly leading to blindness at its final stage. Currently, there is no known treatment or procedure that provides a final cure, even with the use of stem cells or microsensor implants on the retina. Patients with RP have their night vision impaired, as well as difficulty in depth, focus, and clarity as a result of RP and other concomitant diseases such as myopia and cataracts. This paper proposes a technology to support people with Retinitis Pigmentosa. It is a software application for mobile devices that offers specific functionalities, such as, camera zoom, object detection, object tracking, face detection, person tracking, sharper images, and speech interaction. In addition to these, it is possible to obtain information regarding the emotional state with the processing and automatic detection of faces, which enables the carrier of $\mathrm{RP}$ to interact more naturally with the people in his surroundings. The proposed system also enables the mobile device to be used in a smartphone holder acting as glasses, making it a wearable technology. In the proposed solution, the Android Platform with the OpenCV technology was used. Usability tests have been made and the results demonstrated that the proposed technology can be useful and functional. In addition, there are many other possibilities for the use of a smartphone, such as, adding an extra camera as well as motion interfaces. This technology solution is one that can be accessible by many RP users, given the choice of the android mobile platform.
\end{abstract}

\section{General Terms}

Augmented Interfaces, Assistive aid

\section{Keywords}

Retinitis pigmentosa, wearable technology, android, opencv

\section{INTRODUCTION}

Retinitis Pigmentosa is a group of hereditary retinal diseases (inherited diseases) that causes retinal degeneration. They affect the retina, which is the layer of light-sensitive tissue at the back of the eye. As the light-sensing cells in the retina deteriorate vision loss occurs. There are two types of photoreceptor cells: the cones and rods. In most cases of Retinitis Pigmentosa (RP), there is the primary degeneration of the rod cells, which affects the peripheral and night vision. Followed later on by the degeneration of the cone cells, which affects the color perception, sharpness and central vision. Early symptoms in rod-cone dystrophy are night blindness, followed by the progressive loss of peripheral vision. When cone degeneration occurs there will be a decrease in central vision and the loss in perceiving colors. Most cases patients will have their visual acuity preserved in the early and mid stages of the disease [1, 2, 3].

RP patients have a higher total annual health care cost. Since they will consume a higher amount of health services that generally has higher costs [4]. In the recent study they took into account only the health care costs that were captured by insurance claims, not including expenses for caregivers, rehabilitation, home assistance and institutional care. The total annual health care cost was estimated to be $\$ 7317$ higher for RP patients [4].

The use of Augmented Reality (AR) is growing and it helps to bring together the physical and digital world while enhancing important, relevant information. It can greatly assist in many areas, such as helping children with autism stay focused, or even helping drivers see around blind spots [5, 6]. For people that have low vision augmented reality can help gain some of the context-aware data they lost.

This paper presents an AR technology based on mobile devices helping bring specific functionalities, such as, camera zoom, face detection, sharper images, and more to help people on a daily basis. Thus, augmented user interfaces can be useful to better observe the environment by people with the degenerative eye disease. There has been a lot of advancements in technology that can be used to make visual aids which can benefit people who suffer from visual impairment. Nowadays there are advanced smart-phones with powerful processors, high-resolution displays, and highresolution cameras all built into a little device. As well as better and improved digital image processing algorithms. With this it opens many new possibilities to assist those who are visually impaired [7].

\section{RELATED WORKS}

People who are totally or profoundly blind may resort to using the Brainport [8] device or the Argus II [9]. On less severe cases where people have low vision and are still not profoundly blind, they can use eSight [10] or Orcam [11]. All of these assisting devices contain at least one camera and one central processing unit for its purposes. In addition, these solutions are expensive, serve to one specific purpose and are not completely portable. Based on smartphone devices it is possible to propose a low cost aid with similar functionalities compared to $[10,11]$ which can be accessed by multiple people. Also, smartphones can be easily embedded into AR headsets becoming less obstructive, such as wearable devices. 


\section{MATERIALS AND METHODS}

\subsection{Development Process}

In creating a solution for RP users the system requirements needed to be elicited, since it is a complex process and it greatly depended on feedback that needed to be obtained from RP users. In order to do this activity there had to be a lot of interaction with at least one user that had RP disease, to go step by step in analyzing each specific requirement. Consequently, an incremental development process is highly required.

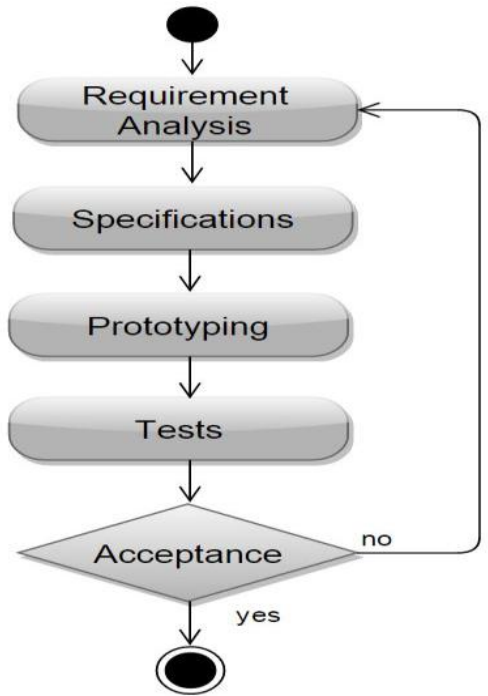

Fig 1: The Development Process

In Requirement analysis there would be a study of the requirements to be worked on each activity, which is an application within the software. The software has different activities with each activity having its own requirements, but they all follow the same development process as presented in Fig. 1. The specifications would be where the planning and design of the interfaces took place, the methods, and parameters that could be implemented to satisfy a requirement.

The prototyping phase was implementing the specifications and constructing the interfaces setting it up for future tests. In testing, a user with low vision would test the device regarding the requirement. If after the evaluation it was able to pass the test there would be no need for further changes in the activity. In case of not being acceptable, then there would be a reevaluation of the process and work once again on improving certain areas based on the user's feedback. After each iteration there would be many positive changes to the activity, gradually improving its results. Most of the development process needed the interaction of a user that had low vision to assist in the evaluation of the activity, giving feedback throughout the process.

\subsection{Technology}

The software has been developed for the android mobile device, since it is a growing platform and has a great amount of users. Developed in Java using the Android SDK with the Eclipse IDE. Needing the minimum version of android 4.0.0 (called Ice Cream Sandwich). The phone used for testing and development was the galaxy S4 active. It comes with an 8megapixel back camera, and has a 1080p high resolution display. Also, a Sony Z3 was used to make fine adjustments and usability testing. Z3 has better hardware capabilities compared to the S4. The software uses the OpenCV library which contains optimized algorithms for image and video analysis. The version used was 2.4.3 throughout the development process. Using OpenCV for image manipulations enabled us to do many things such as, add filters (Canny, Sepia, Gray-Scale), adjust contrast, brightness of video feed, add separate filters, resize images, and even duplicate images in real time. Most tests where made using a Macro 0.67 Wide lens over the phone camera. The camera was able to obtain more visual data of the surroundings using the lens by increasing the visual field the image was able to obtain. This helps in adding more of the scene to the user.

\subsection{Structural Description}

Each software component (Activity) uses the OpenCV library for image manipulations according to its own requirements. As demonstrated on Fig. 2 the activity can access the camera to receive the live feed stream thus doing the necessary manipulations at every frame obtained. The Android voice recognition API for the speech is available on all activities. Choosing to use it can be enabled prior to entering any activity. The voice recognition works regardless of having an internet connection as long as the language pack for offline is available. It uses the microphone hardware whenever activated as seen on Fig. 2. In case Voice recognition is enabled, the current activity will have its sensor manager turned on. This will allow us to detect an event such as a shake, to trigger the voice recognition. The sensor manager uses the accelerometer to detect such event.

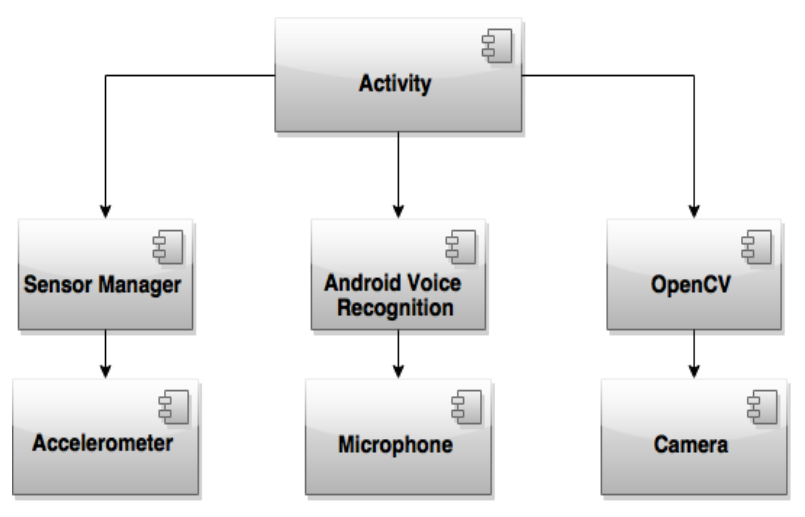

Fig 2: Components

\subsection{Specifications and Prototypes}

The following section demonstrates the different prototypes made along with its improvements. Also, specifying some of the options which are available in the software.

\subsubsection{Void Command Functionalities}

If the Voice Command Feature is Enabled, when the activity is started the sensor manager will remain at a Standby as illustrated in Fig. 3. Whenever the device is shaken, it will prompt the user to speak. After speaking, it will verify the command and check if it is valid. In case the spoken string represents a command then it will execute said command. After executing the command it will once again return to a standby mode. If the command is not valid it will return to standby without executing any commands. 


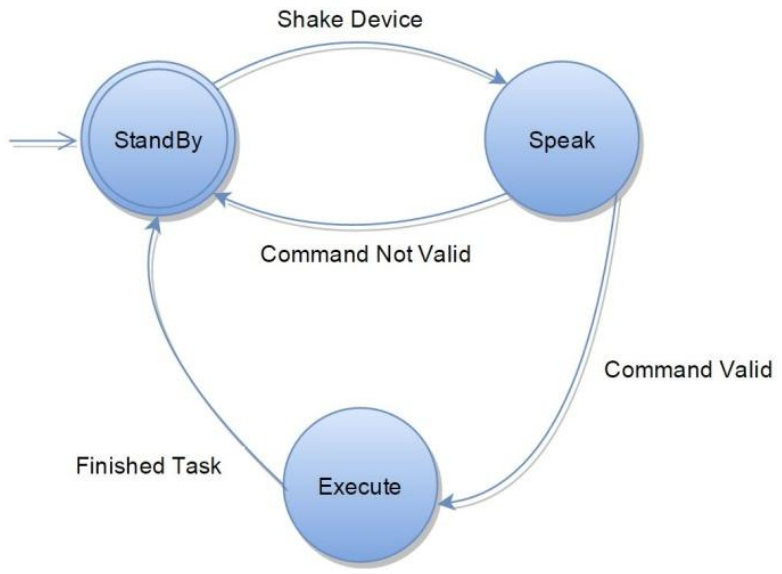

Fig 3: Voice Recognition Process

\subsubsection{Image Filter}

Image filters can be of great help to users who become light sensitive. The use of yellow-orange lenses can help to minimize photophobia [3]. A yellow tint can help to reduce a certain amount of glare. When looking through a phone display the glare will still be visible to the RP user. Therefore, filters were added to the software. After some experiments the sepia camera filter would help to reduce the amount of glare in the display as seen in Fig. 4. This improved the experience of a user who had low vision and was light sensitive to better utilize the device. During the tests the sepia filter was used because the volunteer was very light sensitive and greatly benefited from the sepia; however, the software can be adjusted with different filters.

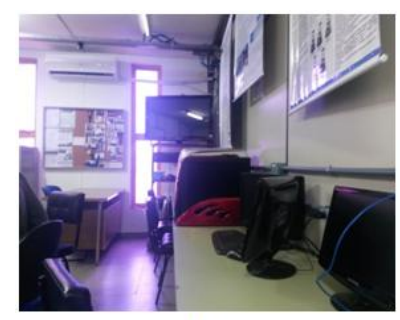

(a)

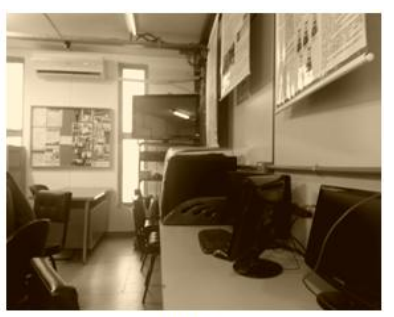

(b)
Fig 4: A sample screenshot. (a) No image filter. (b) Sepia filter used to decrease glare

\subsubsection{Contrast and Brightness Control}

Adjusting the contrast or brightness helps to balance out some of the images to adequately help the viewer ensure the best image for their eyes. The lowest parameters for contrast and brightness can be seen in both Fig. 5a and Fig. 5c. The highest parameters would be shown in Fig. 5b and Fig. 5d. The user is allowed to adjust it to his preferences. For the following tests, the RP user had previously calibrated his preferences for contrast and brightness. In addition, during the development process the RP volunteer user only has 10 degrees of vision left with a good central vision. All activities have the option for Contrast and Brightness adjustments.

\subsubsection{Edges Central Vision Functionality}

The First Prototype can be seen in Fig. 6a where the use of a canny filter to help display edges was evaluated. The purpose of this test was to be able to find a way to show edges and contours to help a RP user with low vision be able to easily identify any obstacle such as a door, table, or chair that might obstruct his path. The canny filter helps us to find contours and edges in a given image. After some iterations the image was divided into 4 peripheral views and a central vision as demonstrated in Fig. 6b. This helps the user to easily focus ahead, while looking at the peripheral in case any line comes crossing to indicate a possible obstacle. Using the Wide Lens RP users were able to widen the field of view, thus allowing the user to have a bigger central view. This had a huge impact on the activity, as it gave him more contextual data of his environment.

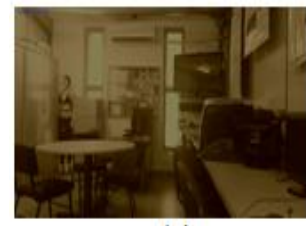

(a)

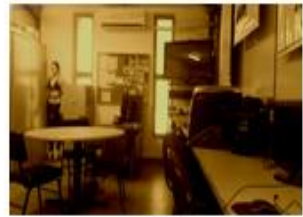

(c)
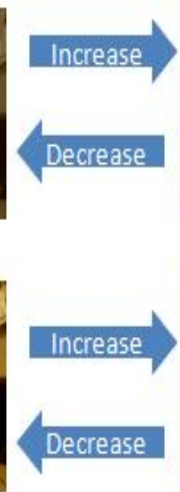

Decrease

.
Fig 5: Contrast and Brightness Adjust. (a) Low contrast. (b) High contrast. (c) Low brightness. (d) High brightness

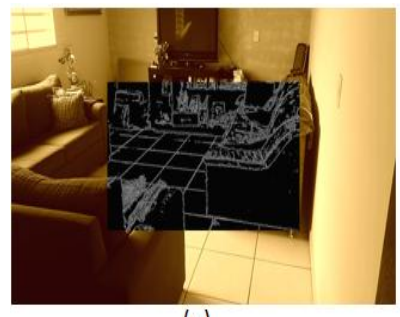

(a)

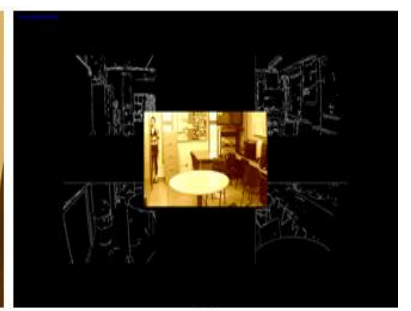

(b)
Fig 6: Edges Central Vision Prototypes. (a) Beginning Stages. (b) Advanced Stages

\subsubsection{Peripheral Central Vision Functionality}

The purpose of this functionality was to bring the peripheral vision closer to the central vision. Allowing the user to have a wider view. With a small glance the user should be able to see what's in each respective corner with little effort. In the intermediate prototype each square represented a portion of the full image and resized to fit their sizes as seen on Fig. 7a. The image did not seem consistent as there were distortions so improvements needed to be made. In the intermediate prototype testing the user found that the middle needed to expand and the corners were too far apart as seen on Fig. 7.

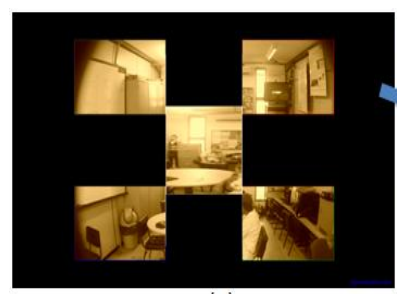

(a)

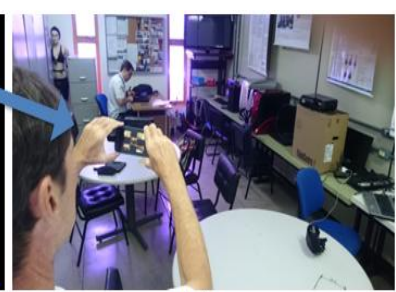

(b)
Fig 7: Peripheral Central Vision. (a) Screenshot of the camera view. (b) User testing

After multiple iterations and adjustments the corners now displays $1 / 4$ of the original image in regards to their location. 
In addition, the volume key can be used to increase or decrease the visual data on the central rectangle. This range varies from $70 \%$ to $30 \%$ of the original full image from a central point. In other words, it is like cropping the image from the center. The user can adjust how much he will see in the center, while having the corner rectangles show their respective corners of the image. In Fig. $8 \mathrm{a}$ and $8 \mathrm{~b}$ the center image displays about $70 \%$ of the original image. Using the Wide Lens with $70 \%$ view it helps to obtain more of the scene as demonstrated in Fig. 8b. Making adjustments in the center view while using the volume key the amount of view inside the central area was decreased to a minimum of $30 \%$ of the original image from a central perspective. The results can be seen on Fig. $8 \mathrm{c}$ and $8 \mathrm{~d}$, while Fig. $8 \mathrm{~d}$ shows the difference when using the wide lens at $30 \%$.

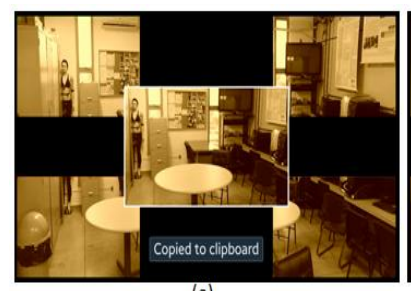

(a)

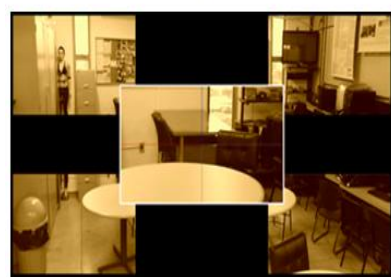

(c)
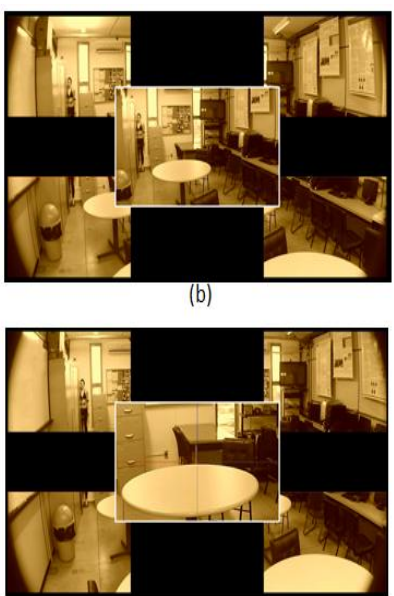

(d)
Fig 8: Peripheral Central Vision where the center rectangle contains an amount of the full image. (a) $70 \%$ Center. (b) $70 \%$ Center with Wide Lens. (c) $30 \%$ Center. (d) $30 \%$ Center with Wide Lens

\subsubsection{Enhanced View Functionality}

The purpose of this functionality was to be able to enhance visual details for anywhere on the screen, while also being able to bring peripheral details to the center. Broadening the users vision view. The zoom feature helps in enhancing visual details during a live feed. As demonstrated in Fig. 9 the center would be the area selected for amplification, while on the left side the result was displayed.

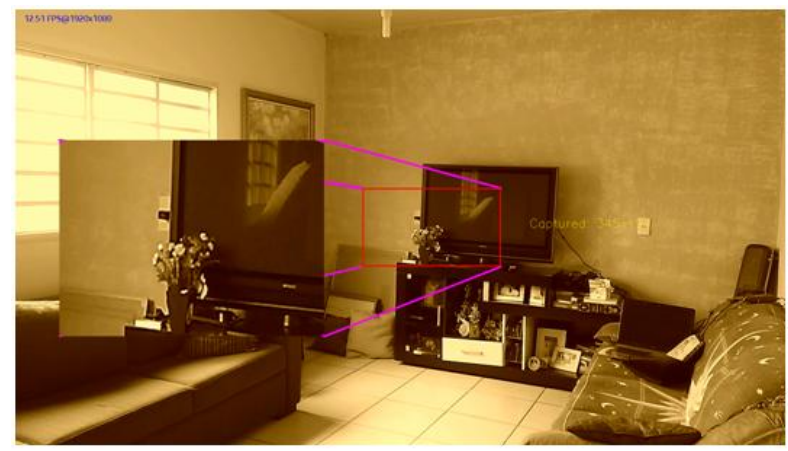

Fig 9: Enhanced View Activity Intermediate Prototype

After multiple iterations and adjustments the red rectangle is the selected region to be enhanced. It can be moved to anywhere in the screen and also increase or decrease the area of interest within certain limits by using the volume key as shown in Fig. 10. Contrast and Brightness options are also available in the options menu. There is also the option to increase or decrease the size of the center region as shown in Fig. 11.

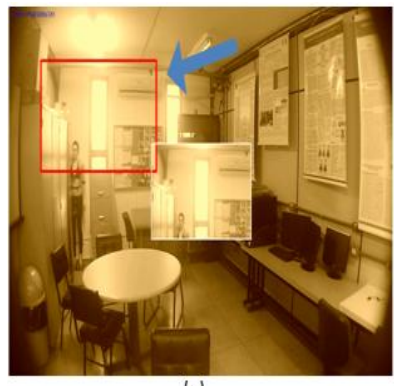

(a)

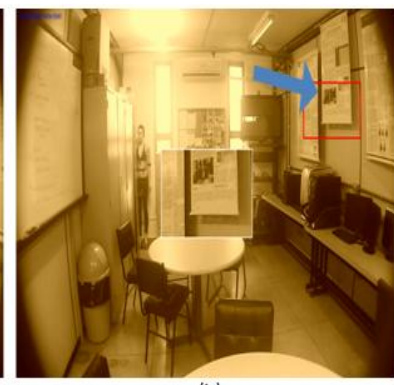

(b)
Fig 10: (a) Larger Selected Region. (b) Smaller Selected Region

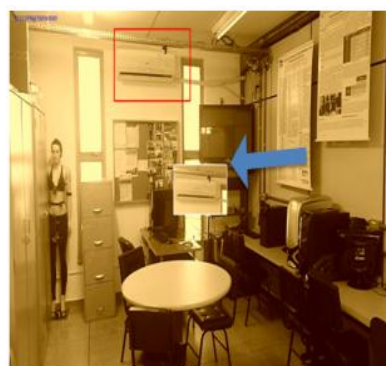

(a)

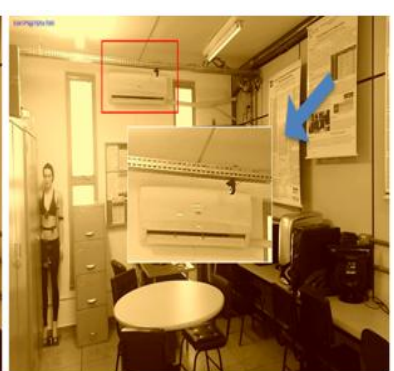

(b)
Fig 11: (a) Smaller Central Region. (b) Larger Central Region

\subsubsection{Headset View Functionality}

Using the head mounted display(smartphone holder), allows us to place the mobile and select the headset view activity. This will change the display as illustrated on Fig. 12 the earlier prototype. The purpose of this activity was to see if using a head mounted display could amplify/increase a users vision field. Which in turn would allow him to see more content at lower quality. After some iterations in the development process, the user can adjust the horizontal position of each separate view by just tapping on the desired screen location for the respective rectangle as seen on Fig. 13.

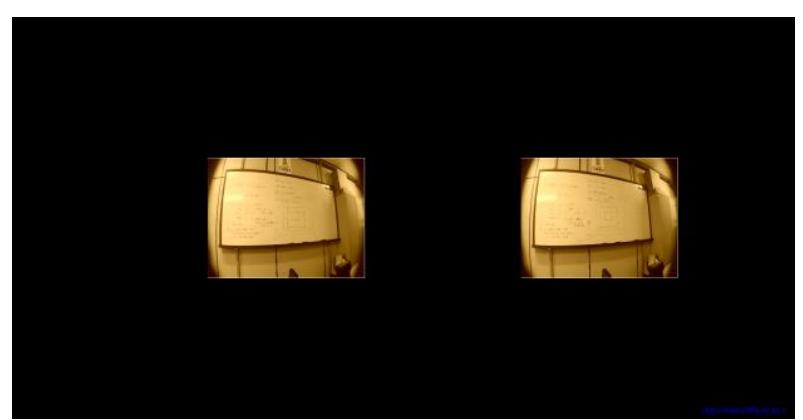

Fig 12: Headset View Activity Early Prototype

In addition, it is possible to decrease or increase the size of the view using the volume keys as well as adjust the contrast and brightness via the menu options. In the early user testing there were various adjustments made as well as feedback obtained from multiple tests as seen on Fig. 14. 


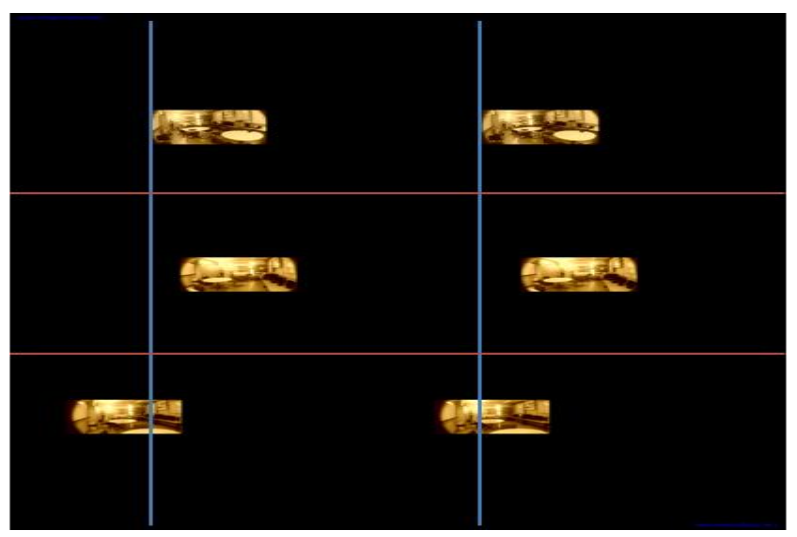

Fig 13: Displaying Different Positioning For Squares. Red line separates different screenshots. Blue line is used as a reference in positioning

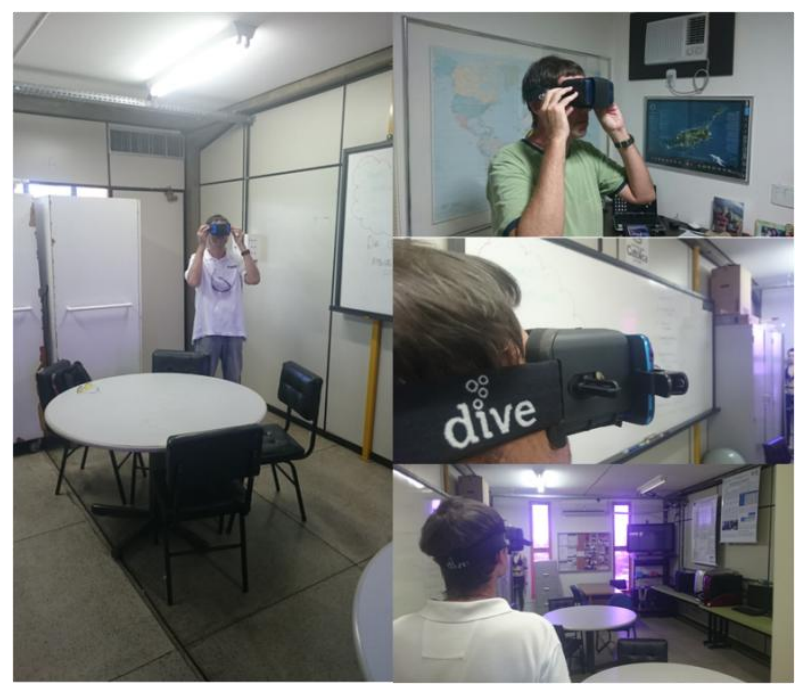

Fig 14: Headset View Early User Testing's

\subsubsection{Emergency Care}

In case of emergencies the device can help send a distress emergency signal calling an emergency contact, or an ambulance for instance. Obtaining the location of the accident through the GPS and sending it out for faster assistance. The system can be connected to sensors and help in monitoring the user. In case drastic changes occurs with the sensor values, the system can act as demonstrated in Fig. 15. The system will be at a monitoring mode, and will ask the user if he is okay in case something happens. With a positive response such as a yes, the system will go back into monitoring mode. In case there is a timeout or a negative response as in the user is not okay, the system can then send an alert either through SMS or an emergency call. Notifying emergency contacts or calling an ambulance. A scenario is illustrated in Fig. 15b where a user had an accident and was then prompted by the system for his condition.

\section{USABILITY TESTS}

\subsection{Face Recognition Counter and} Attention Meter

The face recognition counter is a system's functionality whose purpose is to inform the user of how many different people are looking towards him. Using the openCV face detection a counter was enabled to be constantly counting and alerting the user for any possible faces looking his way as shown in Fig. 16. The number of faces is displayed on the right side of the image. The alerts are given to the user through the speaker by utilizing the mobiles text-to-speech resource.

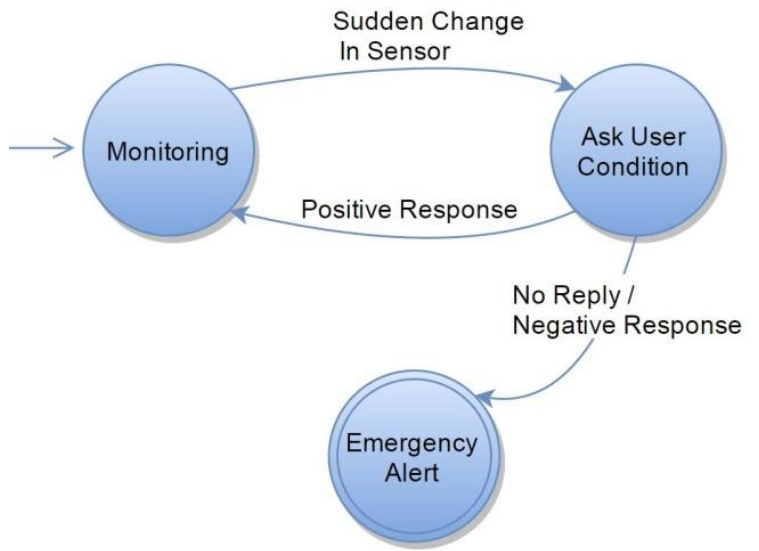

(a)

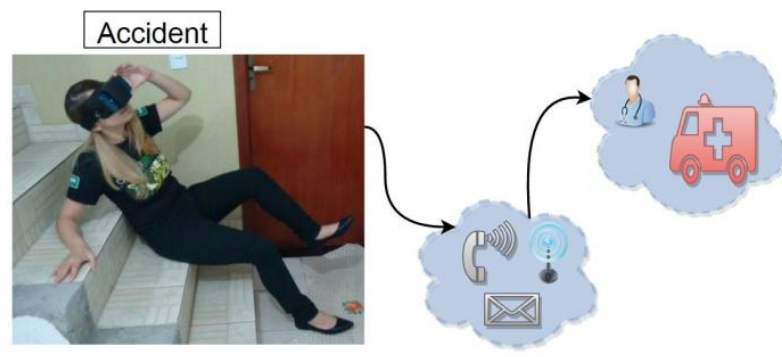

(b)

Fig 15: Emergency Care Feature. (a) The emergency process that monitors a user. (b) Scenario of an accident using the feature following the process above

The attention meter developed at MIT Media Lab [12] was adapted and brought to the android platform devices [13]. It was included in this project whose purpose is to measure a user's attentive level. It is able to identify a person and soon after starts to measure the attention level of the individual as long as they look towards the camera as seen on Fig. 17. This is very helpful in allowing the user to know who is possibly paying attention to him increasing his social interaction. As well as being able to increase his amount of context information.

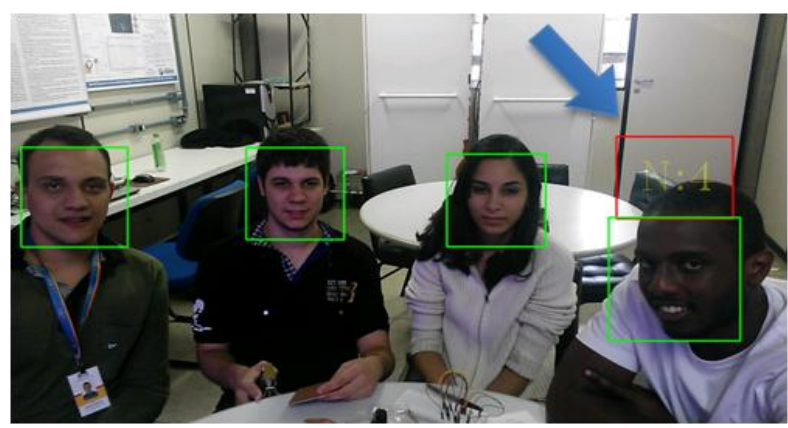

Fig 16: Face Counter Preview 


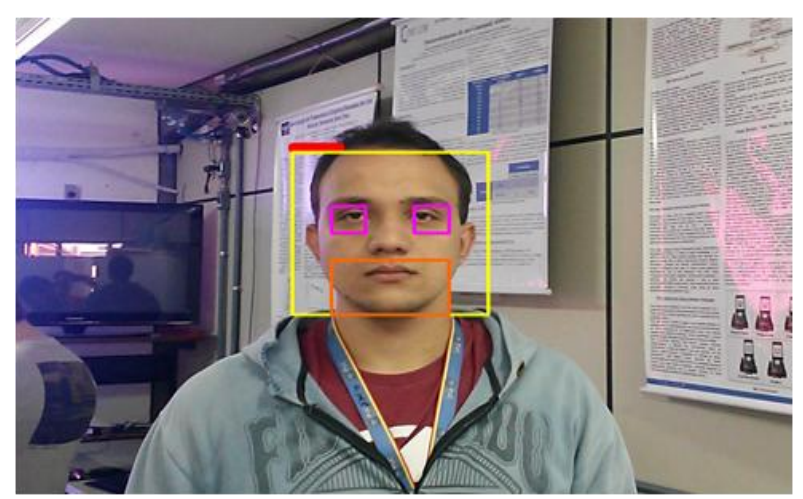

Fig 17: Attention Meter For Mobile

\subsection{Edges Central Vision User Testing}

The activity after improvements has shown to be very functional. The user found it natural to look at the peripheral corners and was able to identify possible obstacles through the canny vision as shown in Fig. 18. This activity is great for individuals who have very low field of vision. As they can focus on the center and sometimes wander the peripherals to see any lines for possible danger obstacles. Future Improvement for this activity is to be able to increase the width of the edge lines.

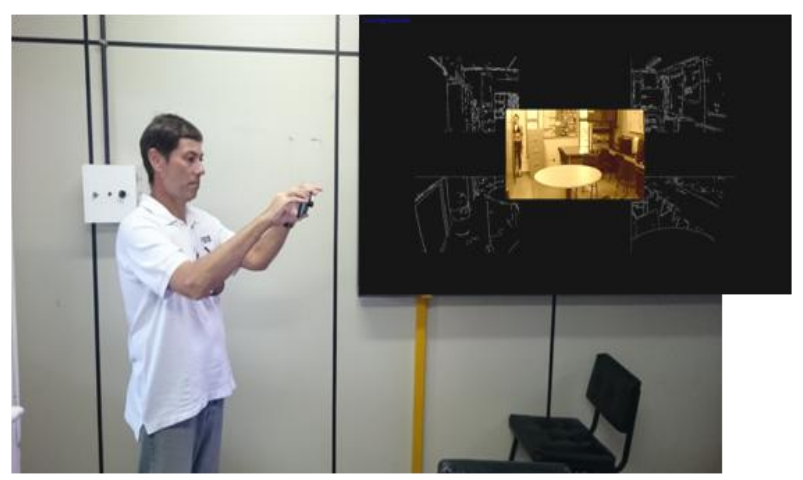

Fig 18: The Edges Central User Testing

\subsection{Peripheral Central Vision Testing}

The results from user testing in this activity were extremely good. The central vision having the option to adjust the amount of view in the center was a success. There was no apparent distortion in the view, and the user found it very easy to navigate in a room as presented on Fig. 19. Using a glance he could look at the image corners and easily assimilate it with the peripheral. This helped bring more visual details to the center of his vision. Keeping in mind that the user only has $10^{\circ}$ of vision.

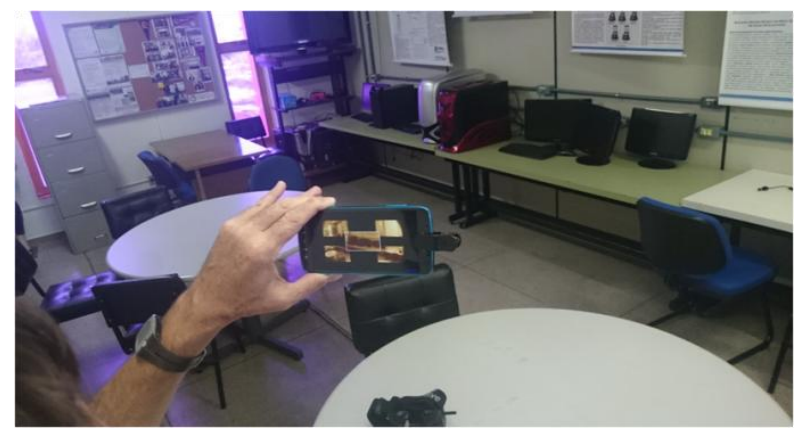

Fig 19: User Testing Peripheral Activity

\subsection{Enhanced View User Testing}

This activity showed great promise. It had very good results, showing that it can be useful and functional. The customizable zoom, and the complete freedom to enhance, magnify, and adjust the image to where you want was a great success as presented on Fig. 20. The functionality was able to bring the details of the peripheral into the center. Depending on the users preference the center could be made to enhance or shrink the view for the selected area.

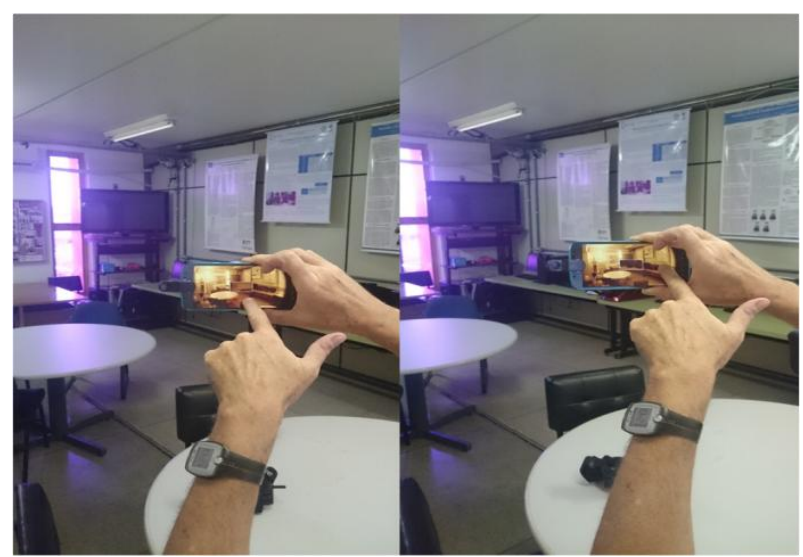

Fig 20: User Testing Enhanced View Activity

\subsection{Headset View User Testing}

The results of this activity was very satisfactory. Figure 21 illustrates the testing environment used to perform a customized version of the Goldmann field exam [14] enabling us to do a basic measure of the user's visual field, mapping the peripheral vision according to ophthalmologist tests.
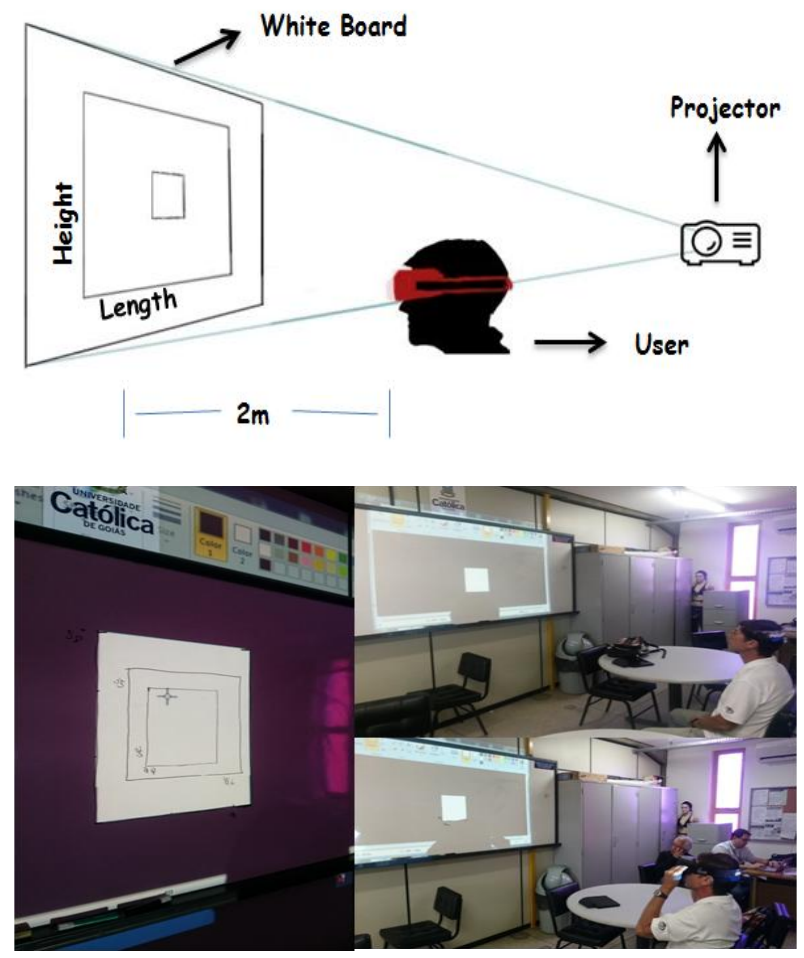

Fig 21: Headset Testing Environment

The user was 2 meters away from a white board and a projector was placed behind him. For better viewing the lights were turned off. In this test scenario there were three different 
test cases as shown in Table I. The first case was measuring the visual field without the headset (normal case). The second case with the user using the headset, and the third case being the use of the headset along with the wide lens. The same methods were used to find the field of view limits for each test case. First, the user had to focus on the middle of the board. After he focused, we would try to find the upper, left, right, and bottom limits separately using the projector. After those limits were found, measures would be taken for each rectangle. This was done for a total of 30 times, doing 10 different measures for each case. This procedure had the aim to verify if any substantial differences between the measures could affect the tests results and to review statistical variables. Therefore, it was possible to obtain a good average for each test case. Using the average length and height, the rectangle area was calculated representing the user's vision field. As demonstrated on Table 1, using the head mounted display along with wide lenses, there was an increase in the field of view of this particular user up to five times as much. Showing that there is a promising future for this type of assistive aid.

Table 1. Headset Viewing Measures

\begin{tabular}{|c|c|c|c|}
\cline { 2 - 4 } \multicolumn{1}{c|}{} & \multicolumn{3}{c|}{ Cases } \\
\cline { 2 - 4 } \multicolumn{1}{c|}{} & $\begin{array}{c}\text { Normal } \\
\text { (no headset) }\end{array}$ & Headset & $\begin{array}{c}\text { Headset and } \\
\text { Lens }\end{array}$ \\
\hline $\begin{array}{c}\text { Length } \\
\text { x } \\
\text { Height }\end{array}$ & $22 \mathrm{~cm} \times 25 \mathrm{~cm}$ & $36 \mathrm{~cm} \times 35 \mathrm{~cm}$ & $47 \mathrm{~cm} \times 58 \mathrm{~cm}$ \\
\hline $\begin{array}{c}\text { Area } \\
\text { of } \\
\text { Vision }\end{array}$ & $550 \mathrm{~cm}^{2}$ & $1260 \mathrm{~cm}^{2}$ & $2726 \mathrm{~cm}^{2}$ \\
\hline
\end{tabular}

\section{CONCLUSION}

There is no cure at this time for Retinitis Pigmentosa so there is a need to rely on assistive devices. The software presented in this paper has shown that by utilizing a mobile device one can create an extremely useful and functional helping assistant. This software can be used on mobile devices to bring new functionalities to help people on a daily basis. Based on a preliminary search using the IEEE (Institute of Electrical and Electronics Engineers), ACM (Association for Computing Machinery) and Google Scholars database, no technological solution that offers a software with customized functionalities to help people with retinitis pigmentosa was found at the moment.

The main contribution of this paper rely on the requirement analysis. Additionally, a software solution was proposed in order to systematize requirements and it allowed us to obtain feedback. The cost of such assistive aid where the hardware in a phone can be utilized, offers a lower cost to this solution because it will mainly depend on the application. With the choice of the android mobile platform, there are capable phones able to run the software accordingly. Each activity from the software has been worked on through multiple iterations improving one step at a time. However, most calibrations were done with the feedback of one user, and with multiple users there would need to be dynamic calibrations for each.

In the future, hopefully more feedback can be obtained regarding each activity from different RP users, to help calibrate the activities along with its new parameters. One other improvement is making this a part of an ambient intelligence system. Broadening the horizon for this type of assistive aids.

The sepia used in testing helped the particular RP user who was light sensitive. Using this filter it takes the color out of the image giving it a yellowed scene, reducing some of the glare for the test user. The test user preferred the sepia over the normal image even when loosing the color aspect. Testing for different types of filters which can help reduce this glare and still maintain the image as closely to the real one can be a major improvement that will adequately benefit different types of users. Equally for testing new filters that can show no distortions.

In helping display edges the canny filter was the first to be evaluated and showed great benefits. Though, there are fine adjustments that can be made to better improve the image result. Such as, increasing the width of the lines and expanding them towards the user or using them as a reference to help send alerts in overlapping circles for the respective corners. Another improving would be to test different filters that could help in displaying contours and edges differently.

Most of the testing's were done in closed environments, so to test in open environments with the influence of different lighting can help further adjust the systems features. Another improvement is to be able to analyze the battery consumption to define algorithms that can lower the battery usage, prolonging the running time of the system.

\section{ACKNOWLEDGMENTS}

This study was supported by FAPEG (in portuguese, Fundação de Amparo à Pesquisa de Goiás) at Pontifical Catholic University of Goiás.

\section{REFERENCES}

[1] Retinitis Pigmentosa. Genetics Home Reference. Online: http://ghr.nlm.nih.gov/condition/retinitis-pigmentosa.

[2] Retinitis Pigmentosa. Lighthouse International. Online: http://lighthouse.org/about-low-vision-blindness/visiondisorders/retinitis-pigmentosa/.

[3] Hamel C. Retinitis pigmentosa.Orphanet $J$ Rare Dis 2006, 1:40. http://www.ojrd.com/content/1/1/40

Online:

[4] Frick KD, Roebuck M, Feldstein JI, McCarty CA, Grover LL. Health Services Utilization and Cost of Retinitis Ophthalmol. 2012;130(5):629-634. doi:10.1001/archophthalmol.2011.2820.

[5] Escobedo, L.; Tentori, M.; Quintana, E.; Favela, J.; Garcia-Rosas, D., "Using Augmented Reality to Help Children with Autism Stay Focused," Pervasive Computing, IEEE, vol.13, no.1, pp.38,46, Jan.-Mar. 2014 doi: 10.1109/MPRV.2014.19

[6] Tachi, S., Inami, M. and Uema, Y. "Transparent Cockpit: Visual Assistance System for Vehicle Using Retroreflective Projection Technology", in Proc. 2008 IEEE Conference on Virtual Reality, pp. 185-188, Reno, USA (2008.3)

[7] The Lasker/IRRF Initiative for Innovation in Vision Science (2014) Chapter 7- Restoring Vision to the Blind: Advancements in Vision Aids for the Visually Impaired. Trans. Vis. Sci. Tech.: December 2014, Vol. 3, No. 7. 
[8] Arnoldussen, A., \& Fletcher, D.C. (2012). Visual Perception for the Blind: The BrainPort Vision Device. Retinal Physician, 9(1), 32-34.

[9] The Argus® II Retinal Prosthesis System. Second Sight. Online: http://www.2-sight.com/argus-ii-rps-pr-en.html.

[10] ESight Corp. Home - ESight. Home - ESight. Online: http://www.esighteyewear.com/.

[11] OrCam, 2013. Online: http://www.orcam.com/.

[12] Chia-Hsun Jackie Lee, Jon Wetzel , Ted Selker, Enhancing interface design using attentive interaction design toolkit, ACM SIGGRAPH 2006 Educators program, July 30-August 03, 2006, Boston, Massachusetts.

[13] Paula, B. "Uma Implementação do Algoritmo Attention Meter para Dispositivos Android.", 2014. Bachelors Thesis in Computer Engineering. Pontifícia Universidade Católica de Goiás.

[14] A.D.A.M. Medical Encyclopedia [Internet]. Atlanta (GA): A.D.A.M., Inc.; O2015. Visual Field; [updated 2013 Feb 7; cited 2015 Jun 10]; [about 2 p.]. Available from:

http://www.nlm.nih.gov/medlineplus/ency/article/003879 .htm 